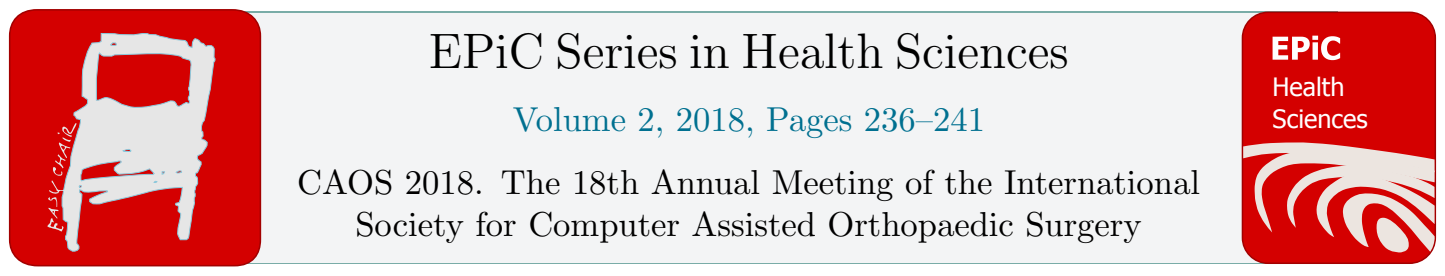

\title{
Application of a novel customized guide template in percutaneous pedicle screw placement: an in vitro study
}

\author{
Su-Hua $\mathrm{Wu}^{1^{*}}$, Guo Zheng ${ }^{3}$, Jian-Hua Chen ${ }^{2}$ and Sheng-Hai Wang ${ }^{2 *}$ \\ ${ }^{1}$ Department of Orthopedics, No.538 Hospital of P.L.A, Hanzhong, shanxi, China \\ ${ }^{2}$ Department of Orthopedics, Hanzhong Central Hospital, Hanzhong, shanxi, China \\ ${ }^{3}$ Department of Orthopedics, Xijing Hospital, The Air Force Medical University, Xi'an, China \\ Wusuhuaczh2@163.com, Wangshorthopedic@163.com
}

\begin{abstract}
In open surgery for spine stabilization, the pedicle screws are often placed using patient-specific guide templates since they can improve the screw placement accuracy and simplify surgery. However, the conventional fit-and-lock template requires full exposure of the bony structures and is therefore not suitable for minimally invasive procedures. In this study, we constructed a novel guide template for percutaneous pedicle screw placement. Its feasibility and the accuracy of template-assisted pedicle screw placement were assessed using an excised calf spine model. As a result, all inserted guide wires and 27 of 30 simulated screws (90\%) were completely in the pedicle. The remaining screws $(10 \%)$ penetrated by less than $2 \mathrm{~mm}$. The average distance and angular deviations of the guide wires were $1.46 \pm 0.60 \mathrm{~mm}$ and $1.10 \pm 0.84^{\circ}$. Our study demonstrate that this novel guide template is technically feasible and enhances the accuracy of percutaneous pedicle screw placement. Moreover, it may simplify the surgery and minimize intraoperative radiation. Further research on its clinical applications is warranted.
\end{abstract}

\section{Introduction}

Percutaneous pedicle screw placement can helps surgeons to minimize damage to the dorsal musculoligamentous complex [1,2]. This in turn yields better surgical outcomes, including reduced blood loss, shorter hospital stays, and less postoperative pain [3-6]. However, compared with open procedures, it is technically more demanding to place the pedicle screws using the percutaneous approach because of limited visualization of the anatomical landmarks. This technical difficulty can lead to inaccurate screw placement, prolonged operative times, and increased exposure to radiation, especially when the surgeon is still in the learning stage [2, 6-8]. Indeed, several studies report that 
percutaneous pedicle screw placement using traditional C-arm guidance results in pedicle screw misplacement in 6.6-30\% of cases. Moreover, some of these inaccurate placements had pathological consequences [8-12]. To overcome this, some surgeons have chosen to use computer-assisted navigation systems or surgical robot systems: these methods indeed associate with better surgical accuracy and reduced radiation exposure [13-15]. However, these systems have not achieved widespread use because of the high cost of the equipment and the long time needed to set up the operating system.

During open surgery for spine stabilization, patient-specific guide templates are often used to guide pedicle screw insertion as they help assure accurate screw placement and thus can significantly simplify and shorten the surgery [16-19]. However, the conventional fit-and-lock template requires full exposure of the bony structures, which naturally obviates its usefulness for minimally invasive procedures. The present study describes a novel guide template that can be used for percutaneous pedicle screw placement. Its feasibility and the accuracy of template-assisted pedicle screw placement were evaluated using an excised calf spine model.

\section{Materials and Methods}

\subsection{Structure of the guide template system}

Briefly, the guide template is designed by first placing two positioning screws in the spinous processes of the vertebrae that will be fixed. The positioning screws are special cannulated screws that are made of medical titanium alloy (Ti6Al4V)(Fig 1B). Subsequently, the spine is imaged by CT and the CT data are used to plan the ideal trajectories and construct the template, which is manufactured from photosensitive resin by 3D printing. An extension rod is then screwed onto the thread on the head of each positioning screw. Thereafter, the guide template is fitted onto the extension rods. Our guide template system thus consists of the two positioning screws (denoted as 1 in Fig 1A), the two extension rods (2), and the guide template (3).

Thus, if the guide template system is to be applied clinically, the positioning screws will first be inserted percutaneously into the spinous processes under local anesthesia before surgery. The CT data of the spine will then be obtained for trajectory planning, template design, and template manufacture. During surgery, the guide template system will be assembled to assist the accurate placement of the guide wires and then the pedicle screws.

\subsection{Experimental procedure}

Five freshly frozen calf lumbar spines (L1-L5) were thawed. In each, two positioning screws were inserted into the spinous processes of L2 and L4 as anchor points for the guide template (Fig 1B). CT data of the spine and Mimics software were used to obtain 3D reconstructions, and the optimal trajectories of the pedicle screw guide wires were determined. The guide template was designed in 3Matic software on the basis of the positioning screws and planned guide wire trajectories and manufactured by $3 \mathrm{D}$ printing.

After guide wire placement, postoperative CT data were used to determine the angular deviations (AD) and distance deviations (DD) of the guide wires. AD was measured as the angle between the axis of the guide wire and the planned trajectory in 3D space. DD was the average distance from the points on the the guide wire to the axis of the planned trajectory. In 3-Matic software, the DD can be easily calculated with the assistance of the tool "distance to curve analysis".

To evaluate how accurately pedicle screws would be inserted if the template guide system was used, insertion of $6.5 \mathrm{~mm}$-diameter pedicle screws was simulated on computer(Fig 2A3, B3). The degree of pedicle-cortex breach by the simulated pedicle screws was assessed as follow: grade $1=$ no 
penetration (screw is completely within the pedicle); grade $2=$ minor penetration (pedicle wall breach of $<2 \mathrm{~mm}$ ); grade $3=$ moderate penetration (pedicle wall breach $2-4 \mathrm{~mm}$ ); and grade $4=$ severe penetration (pedicle wall breach $\geqslant 4 \mathrm{~mm}$ ).

\section{Results}

A visual examination of the specimens showed that all of the inserted guide wires were completely in the pedicle (Fig 2). Moreover, analysis of the simulated insertion of a $6.5 \mathrm{~mm}$-diameter screw along each guide wire showed that, of the 30 screws, 27 (90\%) achieved grade 1 positions. An example is shown in Fig 2A. The remaining three screws achieved grade 2 positions (10\%). An example is shown in Fig $2 \mathrm{~B}$. The average AD and DD of the 30 guide wires were $1.10 \pm 0.84^{\circ}$ and $1.46 \pm 0.60 \mathrm{~mm}$, respectively.

\section{Discussion}

The placement of the percutaneous pedicle screws is a demanding technique: surgeons cannot visualize anatomical landmarks and thus have to rely heavily on fluoroscopic assistance. This increases the exposure of both the patient and medical staff to radiation and prolongs the operative time [6]. In addition, percutaneous pedicle screw fixation associates with a higher rate of screw misplacement than conventional open procedures. Indeed, when Schizas et al. [8] evaluated the accuracy with which 60 pedicle screws were inserted percutaneously under C-arm fluoroscopy guidance in 15 consecutive patients, they observed that the overall rate of CT scan-confirmed screw perforation was $23 \%$ in axial images and $30 \%$ in coronal images. Similarly, a retrospective review of 488 percutaneously placed pedicle screws showed that the overall incidence of misplacement was $23.6 \%$ (11.1\% cortical encroachment and $12.5 \%$ frank penetration)[10].

In this study, a novel template was constructed to assist the placement of percutaneous screws. Before surgery, two tiny positioning screws were inserted into spinous process percutaneously as markers and anchor points of the template. Thus, instead of being put directly on the bone surface, the guide template is fixed on the bone structure through the screws and extension rods, thereby eliminating the need for fully exposure of bony structures. Moreover, the intraoperative radiation could be minimized with this technique. Indeed, in this study, all gudie wires were inserted without the assistance of intraoperative fluoroscopy and none penetrated the pedicle cortex. The misplacement rate of the simulated screw insertion is only $10 \%$, which is comparable to those achieved by imageguided navigation techniques [14].

\section{Conclusions}

Our novel guide template system significantly improved the accuracy of percutaneous pedicle screw placement. Thus, this approach is technically feasible in humans. It may also simplify the surgery while reducing the intraoperative radiation exposure. Its clinical applications should be researched. 


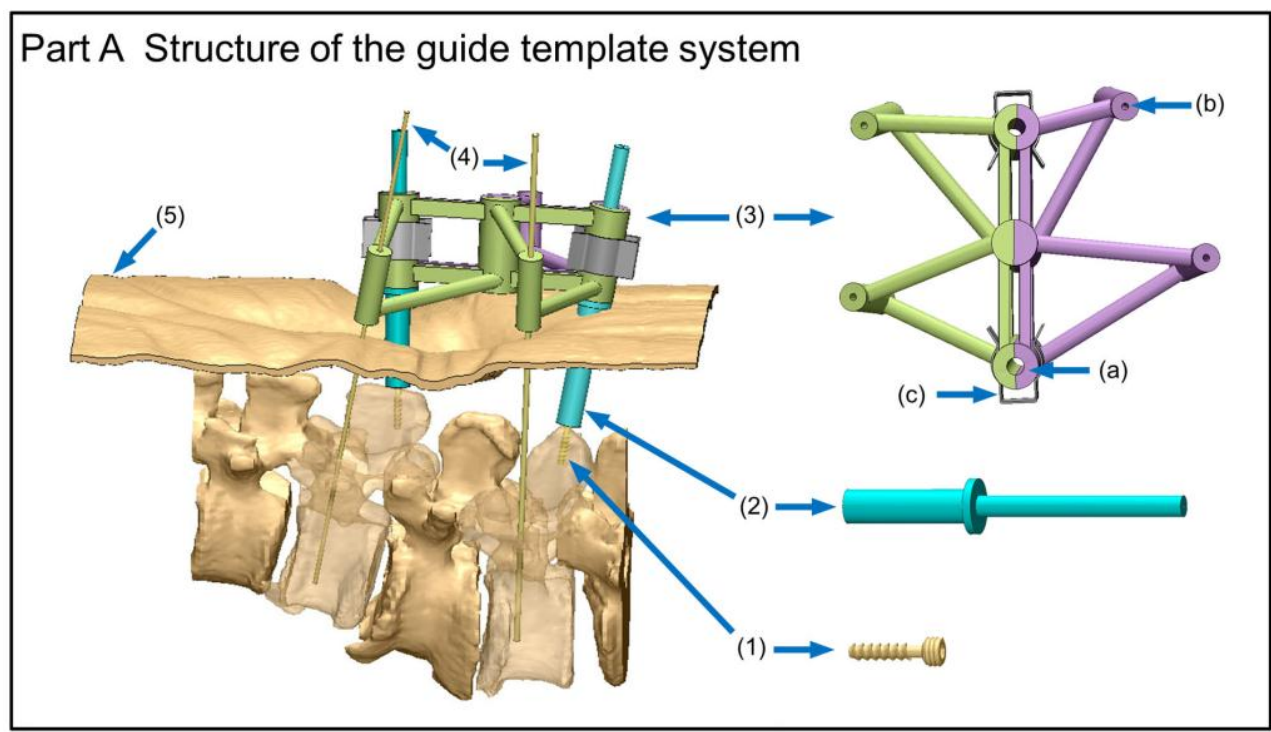

\section{Part B Experimental procedure}
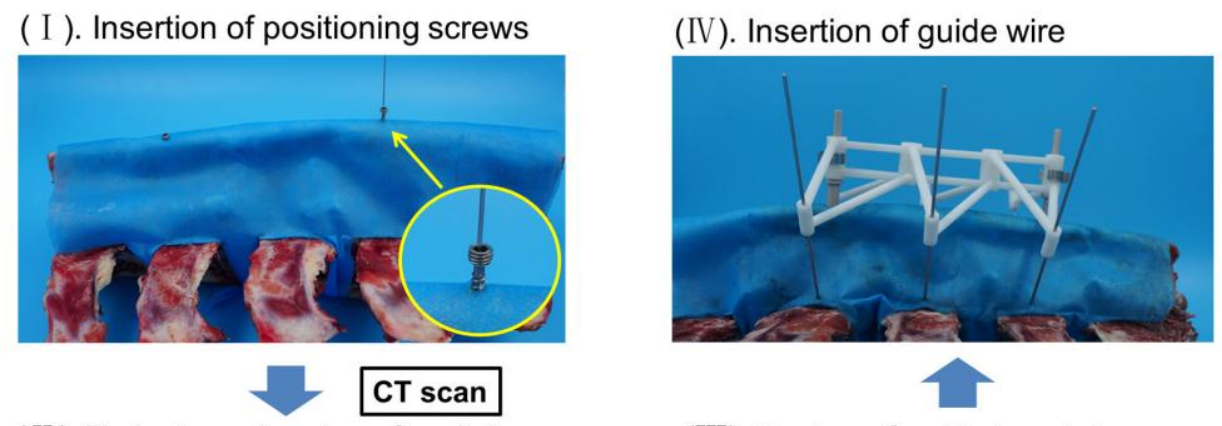

(II ). Trajectory planning of pedicle screw

(III). Design of guide template
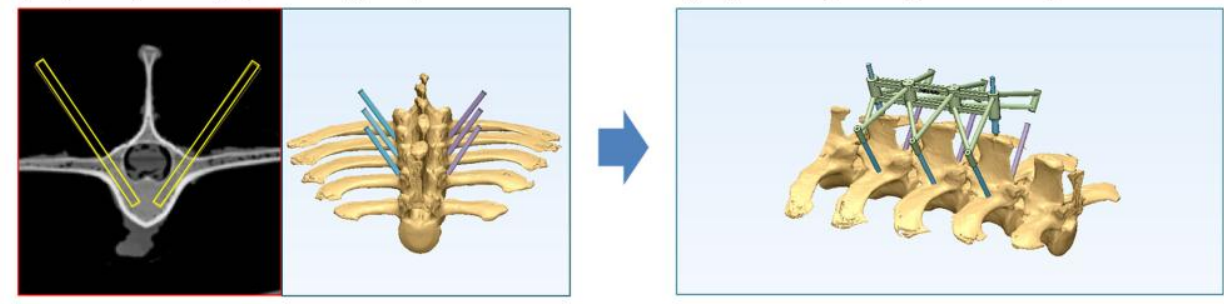

Fig 1 Structure of the guide template system and the experimental procedure. A: The guide template system consists of two positioning screws (1), two extension rods (2), and the guide template (3). The guide template is divided in half for the sake of simplifying installation. Together, it consists of two central fixing tubes (a) and at least four lateral orienting tubes (b). The central fixing tubes are placed around the extension rods, and the two template halves are fixed with metal clamps (c). The orienting tubes, whose trajectories are based on the CT data, are used to guide the placement of the guide wires (4) that will be used to place the pedicle screws later. The skin is designated as (5). B: ( I ) The positioning screws were inserted through the soft cloth covering the lumbar calf spine. ( II ) CT scanning was performed, and a 3D reconstruction of the lumbar spine was generated using Mimics 17.0. The trajectories were shown by the trajectory cylinders. (III) The 3D model of the spine with the positioning screws and trajectory cylinders was transported into 3-Matic Research 9.0, and the guide template was designed. (IV) The template was assembled and the guide wires were inserted through the orienting tube. 


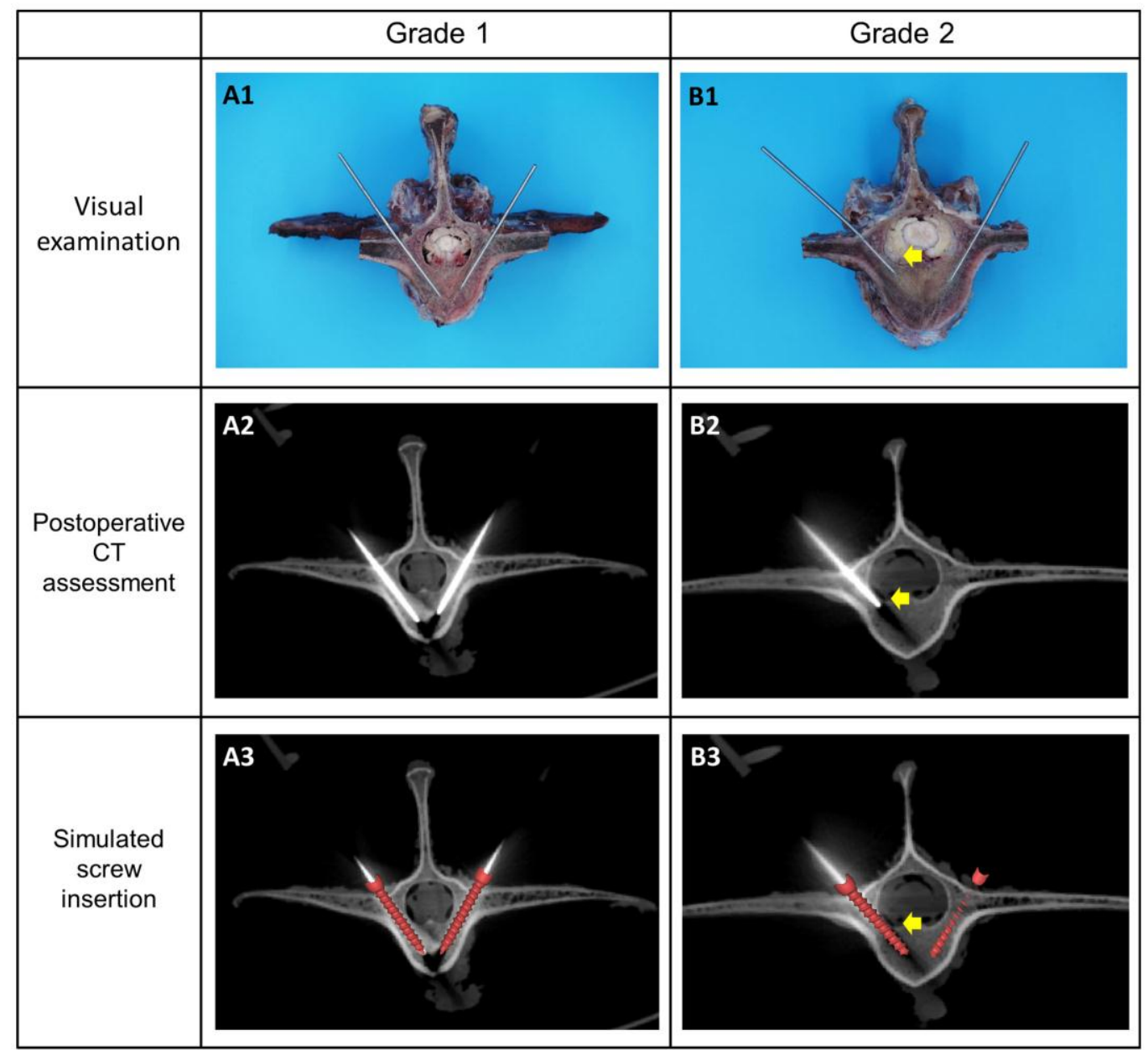

Fig 2 Qualitative evaluation of the accuracy of template-assisted pedicle screw placement. Representative examples of (A) grade 1 vertebrae and (B) grade 2 vertebrae are shown. The vertebrae were sawn in the axial plane after template-assisted guide wire placement and were examined macroscopically (A1 and B1) or were imaged by $\mathrm{CT}$ (A2 and $\mathrm{B} 2$ ). The $\mathrm{CT}$ data were used to generate 3D reconstructions of the operated calf spine, and the insertion of pedicle screws along the guide wires was simulated (A3 and B3). (A) and (B) show an L2 and L4 vertebra, respectively. The yellow arrows indicate grade 2 breach of the pedicle cortex by the simulated pedicle screw.

\section{Reference}

1. Harris, E.B., et al., Percutaneous techniques for minimally invasive posterior lumbar fusion. Neurosurg Focus, 2008. 25(2): p. E12.

2. Yoshida, G., et al., Accuracy of Percutaneous Lumbosacral Pedicle Screw Placement Using the Oblique Fluoroscopic View Based on Computed Tomography Evaluations. Asian Spine Journal, 2016. 10(4): p. 630. 
3. Scheufler, K.M., H. Dohmen, and V.I. Vougioukas, Percutaneous transforaminal lumbar interbody fusion for the treatment of degenerative lumbar instability. Neurosurgery, 2007. 60(4 Suppl 2): p. 203-12; discussion 212-3.

4. Khoo, L.T., et al., Minimally invasive percutaneous posterior lumbar interbody fusion. Neurosurgery, 2002. 51(5 Suppl): p. S166-81.

5. Kotani, Y., et al., Mid-term clinical results of minimally invasive decompression and posterolateral fusion with percutaneous pedicle screws versus conventional approach for degenerative spondylolisthesis with spinal stenosis. Eur Spine J, 2012. 21(6): p. 1171-7.

6. Sidhu, G.S., et al., Minimally invasive versus open posterior lumbar interbody fusion: a systematic review. Clin Orthop Relat Res, 2014. 472(6): p. 1792-9.

7. Sclafani, J.A. and C.W. Kim, Complications associated with the initial learning curve of minimally invasive spine surgery: a systematic review. Clin Orthop Relat Res, 2014. 472(6): p. 1711-7.

8. Schizas, C., et al., Computer tomography assessment of pedicle screw insertion in percutaneous posterior transpedicular stabilization. Eur Spine J, 2007. 16(5): p. 613-7.

9. Nakashima, H., et al., Comparison of the percutaneous screw placement precision of isocentric C-arm 3-dimensional fluoroscopy-navigated pedicle screw implantation and conventional fluoroscopy method with minimally invasive surgery. J Spinal Disord Tech, 2009. 22(7): p. 468-72.

10. Kim, M.C., et al., Factors affecting the accurate placement of percutaneous pedicle screws during minimally invasive transforaminal lumbar interbody fusion. Eur Spine J, 2011. 20(10): p. 1635-43.

11. Wiesner, L., et al., Clinical evaluation and computed tomography scan analysis of screw tracts after percutaneous insertion of pedicle screws in the lumbar spine. Spine (Phila Pa 1976), 2000. 25(5): p. 615-21.

12. Ravi, B., A. Zahrai, and R. Rampersaud, Clinical accuracy of computer-assisted twodimensional fluoroscopy for the percutaneous placement of lumbosacral pedicle screws. Spine (Phila Pa 1976), 2011. 36(1): p. 84-91.

13. von Jako, R., et al., Minimally invasive percutaneous transpedicular screw fixation: increased accuracy and reduced radiation exposure by means of a novel electromagnetic navigation system. Acta Neurochir (Wien), 2011. 153(3): p. 589-96.

14. Fraser, J., et al., Iso-C/3-dimensional neuronavigation versus conventional fluoroscopy for minimally invasive pedicle screw placement in lumbar fusion. Minim Invasive Neurosurg, 2010. 53(4): p. 184-90.

15. Kantelhardt, S.R., et al., Perioperative course and accuracy of screw positioning in conventional, open robotic-guided and percutaneous robotic-guided, pedicle screw placement. Eur Spine J, 2011. 20(6): p. 860-8.

16. Chen, H., et al., Clinical Use of 3D Printing Guide Plate in Posterior Lumbar Pedicle Screw Fixation. Medical Science Monitor, 2015. 21: p. 3948-3954.

17. Fu, M., et al., Construction and accuracy assessment of patient-specific biocompatible drill template for cervical anterior transpedicular screw (ATPS) insertion: an in vitro study. PLoS One, 2013. 8(1): p. e53580.

18. Hu, Y., et al., Deviation analysis of C2 translaminar screw placement assisted by a novel rapid prototyping drill template: a cadaveric study. Eur Spine J, 2013. 22(12): p. 2770-6.

19. Lu, S., et al., A novel computer-assisted drill guide template for placement of C2 laminar screws. Eur Spine J, 2009. 18(9): p. 1379-85. 